\title{
Editorial: Towards Higher Service Performance and Enhanced Security Applying Intelligent Methods and Algorithms
}

\author{
Péter Martinek ${ }^{1}$ \\ ${ }^{1}$ Guest Editor, Budapest University of Technology and Economics, H-1521 Budapest, P.O.B. 91, Hungary
}

Customers demand an ever-growing performance and reliability from every kinds of products today. In the case of modern electronic products this requires both a robust hardware platform and an efficient software based on it. A mobile phone, which is probably one of the most relevant and wide-spread example to the tight coupling of hardware devices and software systems, is expected to provide various services with a great user experience. This means, that working should remain at a low error level or likely error-free and response times should be low even for complex request of the users. On one hand the used hardware devices may determine pure system performance, but applying efficient methods and intelligent algorithms in the software system may increase service performance significantly. Digital technology itself offers multiple methods to detect, correct or even prevent errors originating from hardware malfunctions like applying simply redundancy in the data being stored, transferred or transformed in a digital system. The increasing of available computational capacity also made possible the application of more complex algorithms and methods like artificial intelligence techniques. Artificial neural networks, fuzzy logic based systems, decision trees, etc. facilitates more sophisticated error detection and characterization and help to detect data anomalies and basic correlations. In general, we can say that these techniques may provide a more reliable output for a given input data coming from a database or directly from numerous sensors of a complex hardware system.

System security is also a key issue concerning service performance and user experience. Authentication techniques makes possible to restrict the access to a system and its stored data to a given user or to a group of users. These techniques mostly rely on some secret information or a digital imaging of human biometrics like finger print, palm veins or iris recognition. New algorithms made the biometrics's capture more accurate, the storing more secure and the checking more reliable.

Three-phase induction machines are often used in applications requiring variable speed. The attraction for the combination of static converters with induction motors is because of its robustness, low cost and high performance. However, applied control algorithms can become inefficient when a failure occurs. A paper of this issue presents new neural network based approaches for the diagnosis of the voltage inverter for detecting and locating the IGBT switch open-circuit fault. Machine learning techniques are also applied for modeling economic, social, technical or technological processes. For example, after a suitable training a neural network is able to predict the output for new input parameters. A current paper also shows, how to detect data anomalies and improve the network's performance by sensitivity analysis. With these emerging Artificial Intelligence technology machines may reach a superior level compared to human capabilities in several fields. Nevertheless, making a computer able to understand human language still remains a challenge. Automatic Speech Recognition (ASR) is used to generate transcripts in the process targeting Spoken Language Understanding (SLU). Reader can have an insight in quantifying the semantic bias introduced by ASR error propagation. Analyzing and understanding the appearing of errors and error rate is the first step towards improving reliability in the case of IT services in today's distributed system environment. Hence efficient reasoning about the performance dependability effects of faults is required. The reader can find a new approach to performance and dependability assurance activities. Other papers of the issue present methods to reduce ranging error in GPS-based devices, to create a modern microservices based architecture to increase system's performance, to increase digital information security in a Multi-Biometric Watermarking Scheme and to protect System on Chip architecture against various hardware attacks by applying Hardware Signature Generation.

The articles published in this issue present the latest results of current research fields. The presented new methods and algorithms in various application fields hopefully provides useful information for researchers and professionals either they are interested in this techniques themselves or they have another problem from different field for optimizing performance or increasing security 